\title{
Thermal analysis of PCM containing heat exchanger enhanced with normal annular fines
}

\author{
M. J. Hosseini ${ }^{1}$, M. Rahimi ${ }^{1}$, and R. Bahrampoury ${ }^{2}$ \\ ${ }^{1}$ Department of Mechanical Engineering, Golestan University, P.O. Box 155, Gorgan, Iran \\ ${ }^{2}$ Department of Mechanical Engineering, K. N. Toosi University of Technology, Tehran, Iran \\ Correspondence to: M. J. Hosseini (mj.hosseini@gu.ac.ir)
}

Received: 7 January 2015 - Revised: 4 September 2015 - Accepted: 16 September 2015 - Published: 7 October 2015

\begin{abstract}
In this study, the effect of fins' height and Stefan number on performance of a shell and tube heat exchanger which contains a phase change material is investigated numerically and experimentally. Melting time, solidification time, liquid mass fraction, melting and solidification front and temperature distribution in different directions (longitudinal, radial and angular) are among criteria for the heat exchangers' comparison. In order to generalize the comparison, melting and solidification fronts are studied for different sections of the shell, fin section and mid-section, for different fins' height during charging and discharging processes. The results show that, these two parameters play important roles in the heat exchanger performance. Increasing Stefan number, the melting time reduces; which exhibits a descending trend in rate when the fins are heightened. In addition, investigating both processes, it can be figured out that increasing fins' height influences the solidification time more significantly than melting.
\end{abstract}

\section{Introduction}

Due to the increasing gap between the global energy supply and demand, reaching to a thermally efficient and cost optimized thermal energy storage system has received a considerable attention among researchers. There are three methods for storing thermal energy: sensible, latent and thermalchemical. Among these methods, latent heat thermal storage (LHTS) using phase change materials (PCMs) is known as the most favorable for its high energy storage density with small temperature variation (Mehling and Cabeza, 2007). In other words, PCMs are attractive as they are capable of absorbing and releasing a considerable amount of energy at a nearly constant temperature during melting and solidification processes. Latent heat energy storage systems can be used to store a considerable amount of available thermal energy to be utilized during energy demand period, hereafter providing a promising solution for smoothing the discrepancy between energy supply and demand. Thus, many authors have reported their results of researches on PCM thermal storage during melting and solidification processes in energy storage systems.
Zalba et al. (2003) carried out a review on history of thermal energy storage which deals with phase change materials, heat transfer studies and applications. Sharma et al. (2009) summarized studies on available thermal energy storage systems for different applications.

Temperature and mass flow rate during both melting and solidification are studied for a shell and tube heat exchanger by Hosseini et al. $(2012,2014)$ in which energy absorption capability of the inserted PCM and thermal characteristic of the PCM storage system are investigated respectively. Experimental results indicated that by increasing inlet HTF temperature to $80^{\circ} \mathrm{C}$, theoretical efficiency in charging and discharging processes rises to 88.4 and $81.4 \%$, respectively. They also showed that the same inlet temperature increase leads to $37 \%$ reduction in total melting time.

Ezan et al. (2011) experimentally studied melting and solidification of water as a phase change material in a shell and tube system. They focused on investigation of the effect of flow rate, inlet temperature, thermal conductivity of the tube material and shell diameter on the storage capability of the system. 
From the results obtained, it is concluded that for both solidification and melting processes, natural convection becomes the dominant heat transfer mechanism after a short interval of heat conduction domination. It is also claimed that during discharging period, the inlet temperature of HTF is more effective on the amount of transferred energy in comparison with flow rate, for selected parameters.

Agyenim et al. (2009, 2010, 2011) and Agyenim and Hewitt (2010) investigated melting and solidification of a PCM in a shell and tube heat exchanger with the HTF passing inside the tube and the PCM filling the shell side, for various operating conditions and geometric parameters.

Fins, or more generally extended surfaces, are used to provide additional heat transfer surface in thermal systems. In LHTS systems, various researchers extensively studied the role of different configurations of fins on the performance improvement characteristics of LHTS systems. Subsequently, different numerical studies looking at the impact of fins on overall PCM melting and solidification can be found in literature (Ogoh and Groulx, 2012; Seeniraj and Narasimhan, 2008; Shatikian et al., 2005); typically those studies still neglect natural convection in the liquid PCM phase. Although the cited numerical studies provides the tool to determine optimum fins geometry and LHTS configuration; the defect in natural convection simulations brings about the need to perform experimental studies.

Mosaffa et al. (2012) studied a two-dimensional numerical model based on an enthalpy formulation for prediction of the solid-liquid interface location in a vertical shell and tube LHTS with radial fins. The results indicate that the PCM solidifies more quickly in a cylindrical shell storage than in a rectangular one. In addition the solid fraction of the PCM increases more quickly when the cell aspect ratio is small.

An experimental study of solidification and melting of a phase change material is investigated in a fin and tube heat exchanger by Rahimi et al. (2014). An experimental apparatus was used to investigate the effect of flow rate, inlet temperature and a geometrical parameter (fin pitch) on charging and discharging processes of the heat exchanger. Experimental results showed that, by increasing inlet temperature from $T_{\mathrm{h}}=50^{\circ} \mathrm{C}$ to $T_{\mathrm{h}}=60^{\circ} \mathrm{C}$, melting time decreases more severally in comparison with the same rise from $T_{\mathrm{h}}=60^{\circ} \mathrm{C}$ to $T_{\mathrm{h}}=70^{\circ} \mathrm{C}$.

Mat et al. (2013) conducted numerical study on melting process in a triplex-tube heat exchanger based on three charging cases/approaches with a phase-change material (PCM), RT82. The study focuses on the PCM melting process in a triplex tube in which 3 different heat transfer methods are considered; (1) internal tube is subjected to heat transfer, (2) outside tube is subjected to heat transfer and (3) both sides are subjected to heat transfer. They also studied the effect of internal, external and internal-external fins on heat transfer enhancement between the PCM and the HTF whereas the consequences of fins' height as an effective parameter were investigated. They found that using a triplex- tube heat exchanger enhanced with internal-external fins, melting time reduces to $43.3 \%$ in comparison with the similar finless heat exchanger.

Liu and Groulx (2014) experimentally investigated phase change heat transfer inside a horizontal cylindrical latent heat energy storage system designed with a central finned copper pipe running the length of the cylindrical container, during charging and discharging operations. It was observed that conduction is the dominant heat transfer mechanism during the initial stages of charging, and natural convection dominates once enough PCM melt is present inside the system. However, conduction dominates during the entire solidification process. Complete melting time is strongly affected by the HTF inlet temperature but it reduces to a much less extent by the HTF flow rate.

The effect of axial fins on enhancement during solidification process has also been examined by Ismail et al. (2001). Their experimental measurements and theoretical predictions obtained from their numerical analysis for different fins' heights, numbers of fins, fins' thicknesses and aspect ratios of annular spaces showed that the use of fins improves the heat transfer rate significantly.

Pakrouh et al. (2015) performed a parametric investigation on pin fin heat sink enhanced with a phase change material. Their results show that an increase in number fins as well as their thickness and height leads to a decrease in base temperature while the operating time reduces.

In the present study, melting and solidification of a specific PCM is explored in a finned shell and tube heat exchanger for two fin heights and three Stephan numbers to study the effect of these two variables on some decision making parameters. These criteria include temperature distribution, melting and solidification front and total melting and solidification time.

\section{Geometric details of the heat exchanger}

In order to study the heat exchanger, a double pipe heat exchanger is designed in which fines are properly spaced in the shell and around the tube. The scaled section view of the heat exchanger is exhibited in Fig. 1a to show the internal components and the sections under consideration in color. As can be seen, the mounted and welded 8 fins are spaced equally and symmetrically in a way that the tube axis coincides with the fin's surface normal vector and the sections selected for thermal measurement are introduced in white transparent circular plates. The longitudinal distance of the first fin from the cap is half of the fin spacing.

Figure $1 \mathrm{~b}$ illustrates a photo of the heat exchanger connected to a data logger to record thermal values of the PCM at the considered sections. Complimentary data including the employed materials and shell capacity are given in Table 1.

In order to measure temperature at different positions of the heat exchanger, 3 sections are chosen symmetrically. In each of the sections a number of thermocouples are imple- 


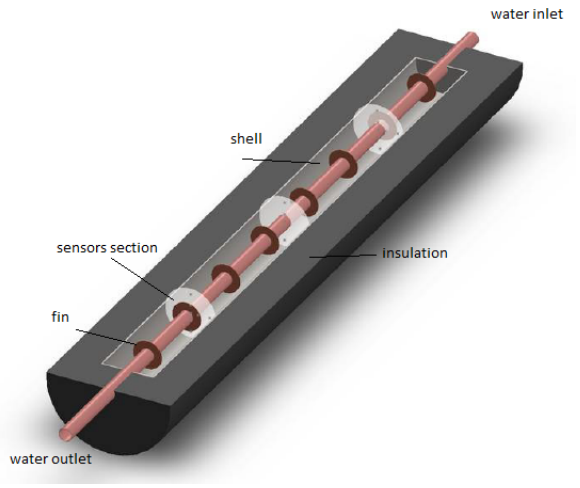

(a)

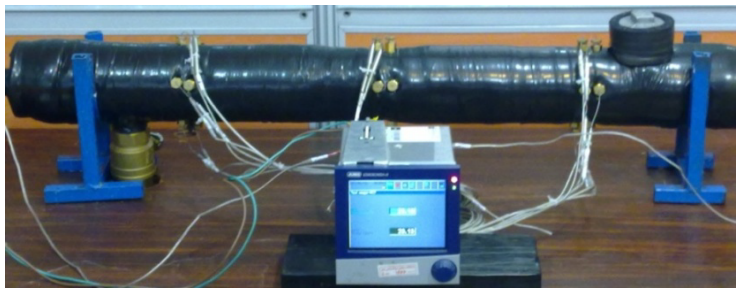

(b)

Figure 1. (a) Section view of the heat exchanger and (b) real photo of heat exchanger.

Table 1. Complimentary specifications of the heat exchanger.

\begin{tabular}{ll}
\hline Specification & Type/value \\
\hline Fins' material & Copper \\
Shell's material & Iron \\
Insulation & Glass wool \\
Number of fins & 8 \\
Shell capacity $\left(\mathrm{m}^{3}\right)$ & 0.005294 \\
Contact surface $(\mathrm{excluding}$ fins $)\left(\mathrm{m}^{2}\right)$ & 0.06912 \\
Small fins height & $13 \mathrm{~mm}$ \\
Small fins' contact surface $\left(\mathrm{m}^{2}\right)$ & 0.02278 \\
Large fins height & $26 \mathrm{~mm}$ \\
Large fins' contact surface $\left(\mathrm{m}^{2}\right)$ & 0.0673 \\
\hline
\end{tabular}

mented. The exact positions of sensors as well as geometric details of the heat exchanger are shown in Fig. 2.

\section{Setup and procedure}

Figure 3 illustrates a schematic diagram of the experimental apparatus which basically consist of a flow control system, the heat exchanger unit and the measurement system.

As the study includes both charging and discharging processes, two different loops are designed to provide hot and cold utility for melting and solidification respectively. The hot loop in which hot bath joins to produce the required heat is connected to the cold loop (which includes a cold bath) utilizing appropriate valve to facilitate the selection of each loop to run the corresponding process. As flow rate is an indispensible component of heat transfer rate calculation, a rotameters is employed in the experimental setup. As observed in Fig. 2, three sections in the shell are designated for thermal measurement; the two end sections include 6 thermocouples while the middle one involves 4 . The exact position of the section as well as angular position and radial distances of every thermocouple is presented in Fig. 2. Considering these
Table 2. Thermophysical properties of RT50.

\begin{tabular}{ll}
\hline Properties & Typical values \\
\hline$T_{\mathrm{S}}[\mathrm{K}]$ & 318 \\
$T_{\mathrm{Liq}}[\mathrm{K}]$ & 324 \\
$\rho\left[\mathrm{kg} \mathrm{m}^{-3}\right]$ & 780 \\
$c_{\mathrm{P}}\left[\mathrm{J} \mathrm{kg}^{-1} \mathrm{~K}^{-1}\right]$ & 2000 \\
$k\left[\mathrm{~W} \mathrm{~m}^{-1} \mathrm{~K}^{-1}\right]$ & 0.2 \\
$L[\mathrm{~J} \mathrm{~kg}$ & $-1]$ \\
$\beta[1 / \mathrm{K}]$ & 168000 \\
\hline
\end{tabular}

16 thermocouples as well as the HTF inlet and outlet thermal measuring thermocouples, 18 thermocouples are incorporated in the experimental setup.

\section{Methodology of tests}

In this study RT50 (Rubitherm $\mathrm{GmbH}$ ) is selected as PCM to be poured within the shell. The most important characteristics of the material are summarized in Table 2. Additional information about the PCM is given by Hosseini et al. (2014).

After the heat exchanger is filled up with liquid PCM and no leakage was observed, a few runs are made in order to calibrate the system. Then the charging process starts, while the solid PCM is at thermal equilibrium with the conditioned lab temperature $\left(23-25^{\circ} \mathrm{C}\right)$. During the charging process, inlet HTF temperature, $T_{\mathrm{H}}$, is maintained at a set temperature using a PID controlled hot water bath. After an imposed $150 \mathrm{~min}$ of charge time, discharging part of the experiment begins. In this process, cold water which solidifies the PCM is pumped from the bath to the heat exchanger. The experiment is finishes after $180 \mathrm{~min}$ of discharge process. During the whole process temperature values are recorded every $1 \mathrm{~min}$.

Using the apparatus and procedures described above, several experiments have been conducted to study the behav- 

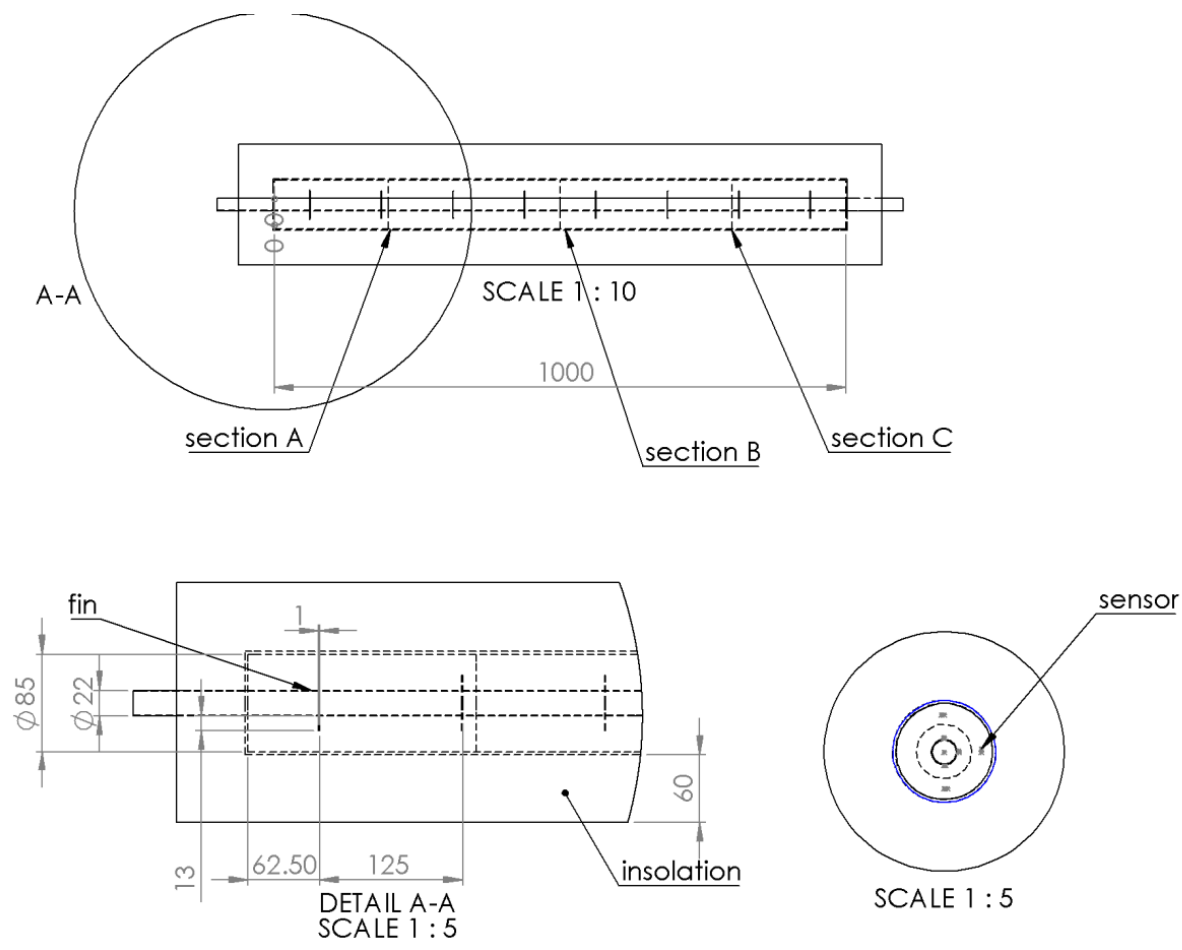

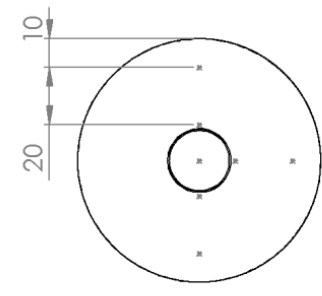

section $\mathrm{A}$

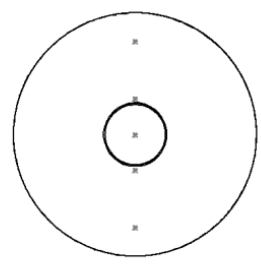

section $B$

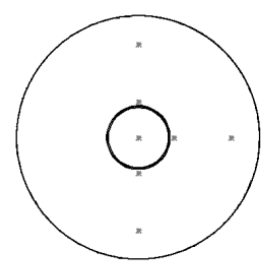

section C

Figure 2. Geometric details of the finned-tub heat exchanger and sensors position.

ior of the PCM during charging and discharging processes. The experiments are performed for different inlet temperatures $\left(70,75\right.$ and $\left.80^{\circ} \mathrm{C}\right)$ of the HTF and fins' height (13 and $26 \mathrm{~mm}$ ). Corresponding Stephan number (Ste) (the ratio of sensible heat to latent heat) as defined in Eq. (1) for each of these hot HTFs are calculated in which the only varying parameter is temperature difference

Ste $=\frac{C_{p}\left(T_{\mathrm{H}}-T_{\mathrm{m}}\right)}{L}$

where $C_{p}, L$, and $T_{\mathrm{m}}$ are respectively the specific heat, the latent heat and the mean melting temperature of the PCM and $T_{\mathrm{H}}$ is the temperature of hot inlet water. For the experiments presented in this paper, the Stefan numbers for the three melting temperatures are $0.26,0.32$ and 0.38 , respec- tively. Discharging experiments are conducted for the same flow rates but only at $T_{\mathrm{C}}=25^{\circ} \mathrm{C}$.

\section{Numerical models}

In order to simulate phase change phenomenon during melting and solidification in a shell and tube heat exchanger, enthalpy-porosity method Brent et al. (1988) and Gong et al. (1999) is used. In the present study, both PCM and water flows are considered to be unsteady, laminar, incompressible and three-dimensional. The viscous dissipation term is considered negligible so that the viscous incompressible flow and the temperature distribution in annulus space are described by the Navier-Stokes and thermal energy equations, respectively. Also dynamic viscosity considered as a func- 


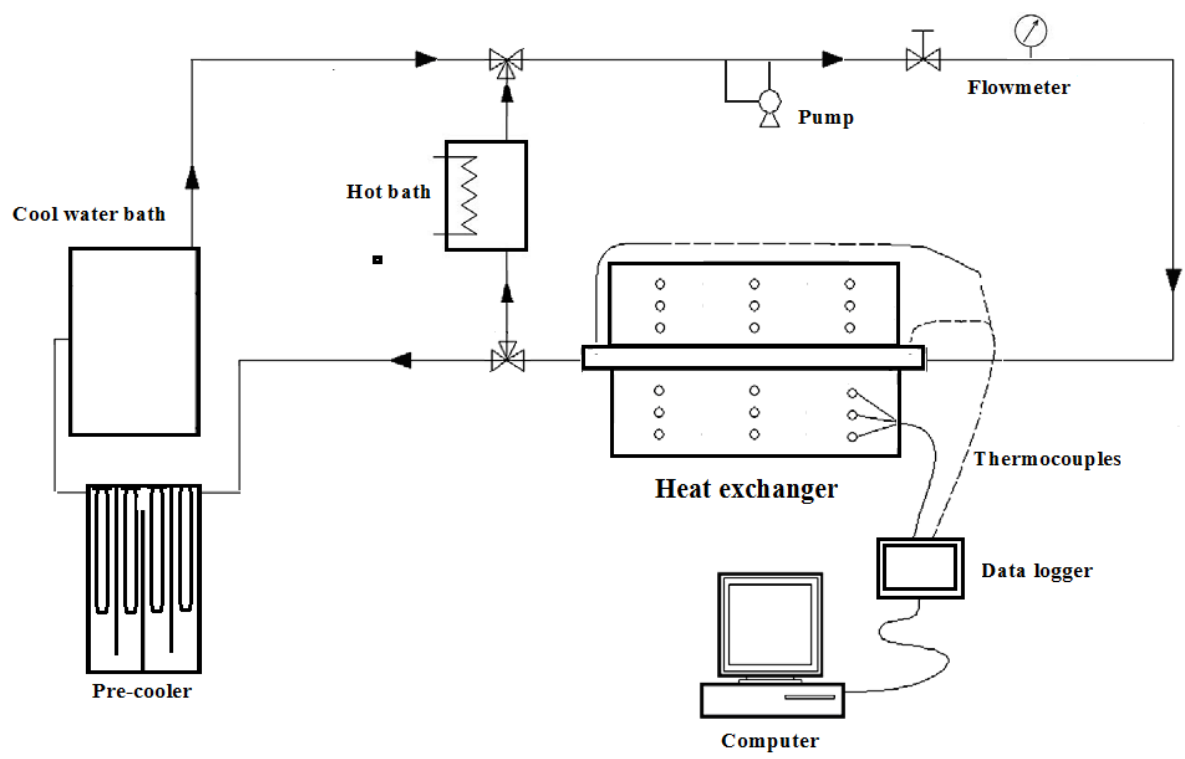

Figure 3. Schematic diagram of experimental setup.

tion of temperature is also used (Hosseini et al., 2014). In addition, the density change within the liquid phase that drives the natural convection is only considered in the body force terms (Boussinesq approximation) in which the variable density is defined as $\rho=\rho_{0}\left(\beta\left(T-T_{\mathrm{Liq}}\right)+1\right)^{-1}$ for $51^{\circ} \mathrm{C}<T<100^{\circ} \mathrm{C}$ in the liquid state. The initial temperature of the whole system is $T_{\text {Ini }}=25^{\circ} \mathrm{C}$. Also the lateral surface of the outer tube is assumed to be insulated. Consequently, the continuity, momentum, and thermal energy equations can be expressed as follows:

Continuity: $\nabla \cdot \boldsymbol{V}=0$

Momentum $: \frac{\partial \boldsymbol{V}}{\partial t}+\boldsymbol{V} \cdot \nabla \boldsymbol{V}=\frac{1}{\rho}\left(-\nabla P+\mu \nabla^{2} \boldsymbol{V}\right.$

$$
\left.+\rho \beta \boldsymbol{g}\left(T-T_{\mathrm{ref}}\right)\right)+\boldsymbol{S}
$$

Thermal energy : $\frac{\partial h}{\partial t}+\frac{\partial H}{\partial t}+\nabla(\boldsymbol{V} h)=\nabla\left(\frac{k}{\rho c_{p}} \nabla h\right)$.

The enthalpy of the material is computed as the sum of the sensible enthalpy, $h$, and the latent heat, $\Delta H$ :

$H=h+\Delta H$

where

$h=h_{\mathrm{ref}}+\int_{T_{\text {ref }}}^{T} C_{p} \mathrm{~d} T$.

The latent heat content can be written in terms of the latent heat of the material:

$\Delta H=\lambda L$

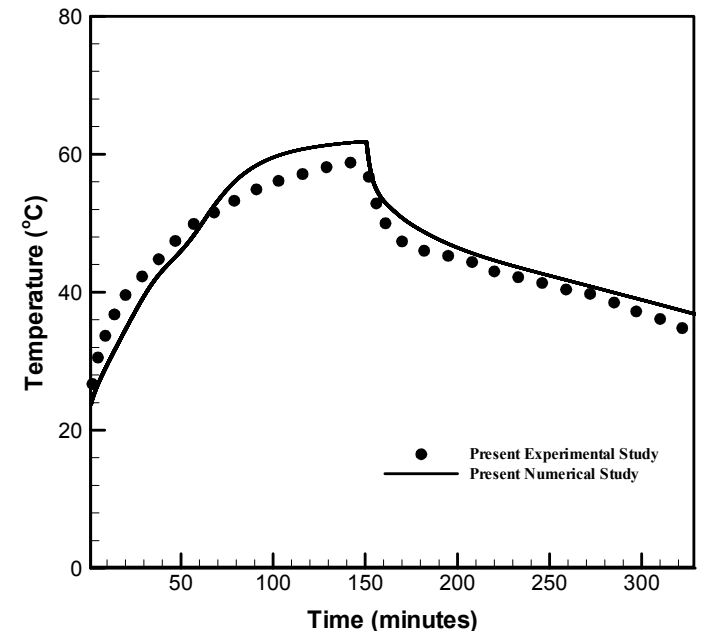

Figure 4. Comparison between numerical and experimental results.

where $\Delta H$ may vary from zero (solid) to $L$ (liquid). Therefore, the liquid fraction, $\lambda$, can be defined as:

$\lambda=\left\{\begin{array}{ll}\frac{\Delta H}{L}=0 & \text { if } T<T_{\mathrm{S}} \\ \frac{\Delta H}{L}=1 & \text { if } T>T_{\mathrm{Liq}} \\ \frac{\Delta H}{L}=\frac{T-T_{\mathrm{S}}}{T_{\mathrm{Liq}}-T_{\mathrm{S}}} & \text { if } T_{\mathrm{S}}<T<T_{\mathrm{Liq}}\end{array}\right.$.

In Eq. (3), $\boldsymbol{S}$ is the Darcy's law damping terms (as source term) that is added to the momentum equation due to phase change effects on convective heat transfer which is defined as: 

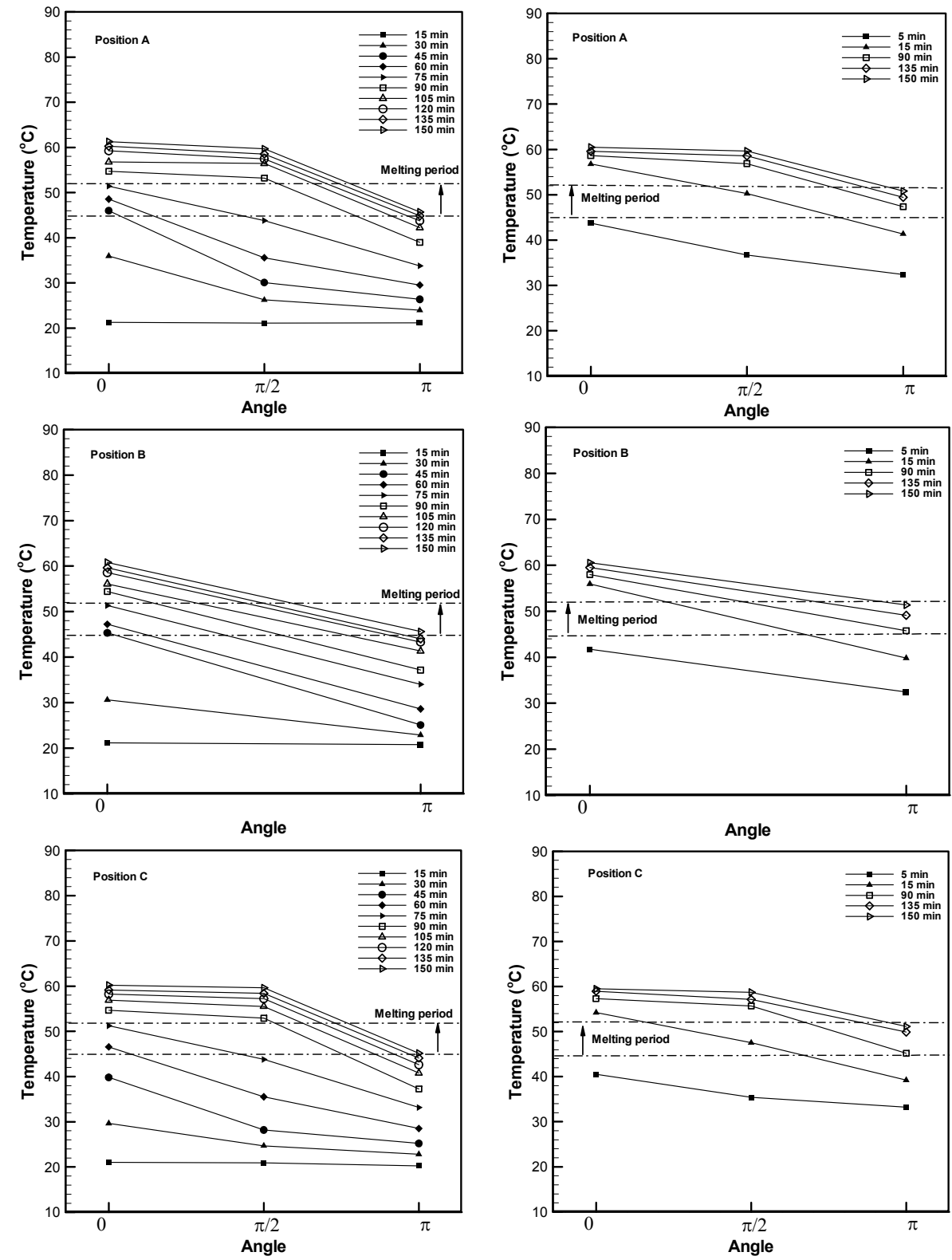

(a)

(b)

Figure 5. Experimental result of temperature distribution for $13 \mathrm{~mm}$ height fins heat exchanger for different angles during charging process for Ste $=0.26$ : (a) $32.5 \mathrm{~mm}$ radial distance and (b) $12.5 \mathrm{~mm}$ radial distance.

$\boldsymbol{S}=\frac{(1-\lambda)^{2}}{\lambda^{3}} A_{\text {mush }} \boldsymbol{V}$

The coefficient $A_{\text {mush }}$ is a mushy zone constant. This constant is a large number, usually $10^{4}-10^{7}$. In the current study $A_{\text {mush }}$ is assumed constant and is set to $10^{6}$.

\section{Computational methodology}

The SIMPLE algorithm (Patankar, 1980) whose task is to couple pressure-velocity governing differential equations and QUICK differencing scheme is employed for solving the momentum and energy ones within a 3-D in-house developed code (Hosseini et al., 2012), whereas the PRESTO scheme is adopted for the pressure correction equation. The underrelaxation coefficient for momentum, pressure, density, melting fraction and thermal energy are $0.7,0.3,1,0.9$ and 1 , respectively. An arrangement of 420000 grids is found to be sufficient for the present study. The time step in the calculations is as small as $0.05 \mathrm{~s}$ and the number of iterations for each time step is 400 . The grid size and the time step were chosen after careful examination of the independency of the 

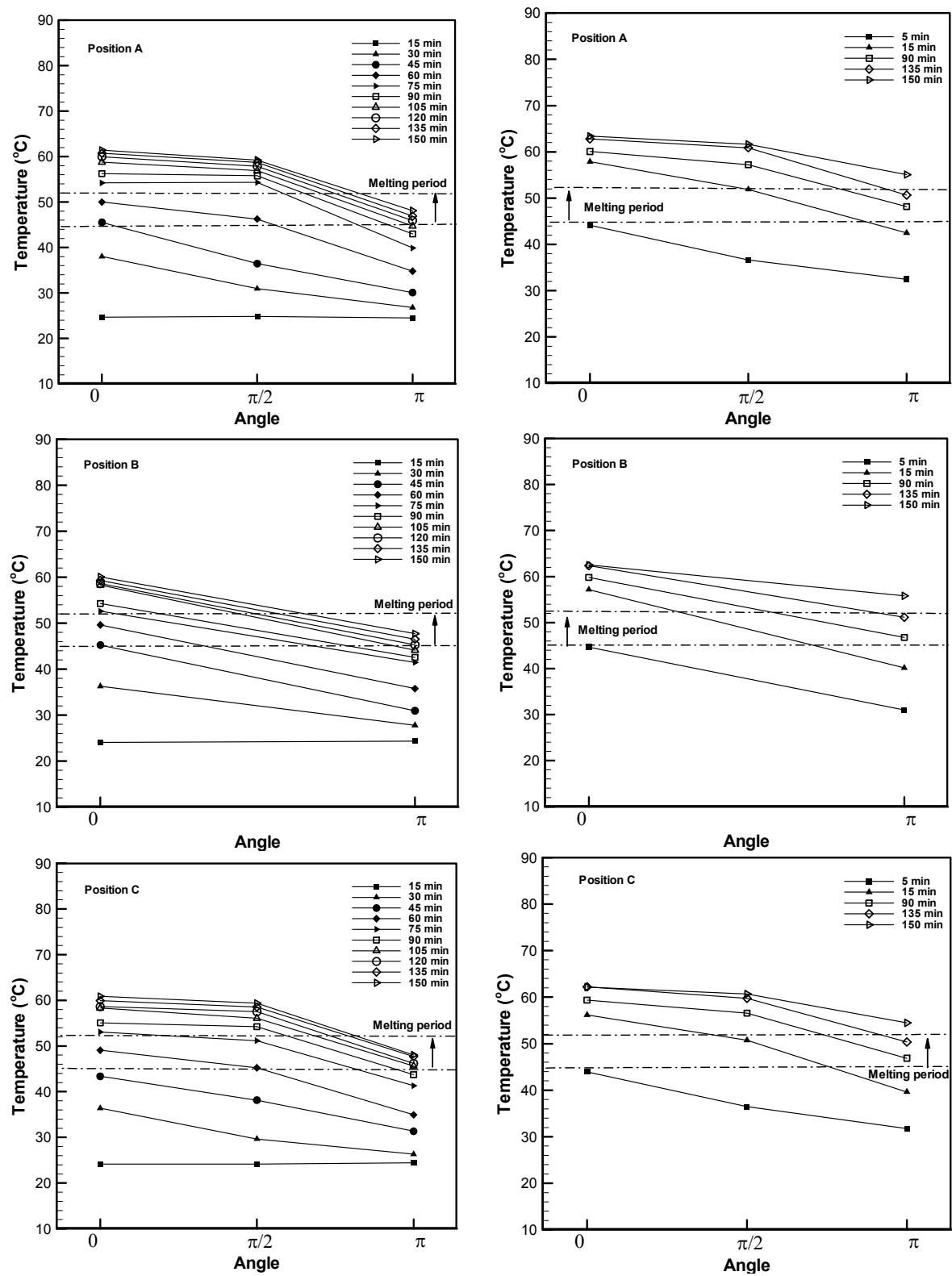

(a)

(b)

Figure 6. Experimental results of temperature distribution for $26 \mathrm{~mm}$ height fins heat exchanger for different angles during charging process for Ste $=0.26$ : (a) $32.5 \mathrm{~mm}$ radial distance and (b) $12.5 \mathrm{~mm}$ radial distance.

results to these parameters. The convergence is checked at each time step, with the convergence criterion of $10^{-7}$ for all variables.

In this section, the presented numerical solution of the simulation of the heat exchanger for fins' height of $13 \mathrm{~mm}$ and Stefan number of 0.26 is validated via the current experiment. Figure 4 illustrates the average temperature of the PCM versus time during charge and discharge processes. The figure exhibits a rational compatibility between these two.

\section{Results and discussion}

\subsection{Charging process}

As a phase change material is exposed to heat, the melting front appears and develops in a way that the ratio of solid PCM to liquid PCM continuously varies while heat transfer mechanism differs for both fronts; the dominated mechanism of heat transfer in the solid region is conduction through which heat is absorbed from the liquid melt and leads to a rise in the solid PCM temperature. In the other region (melt), convective heat transfer accompanying buoyancy based vor- 


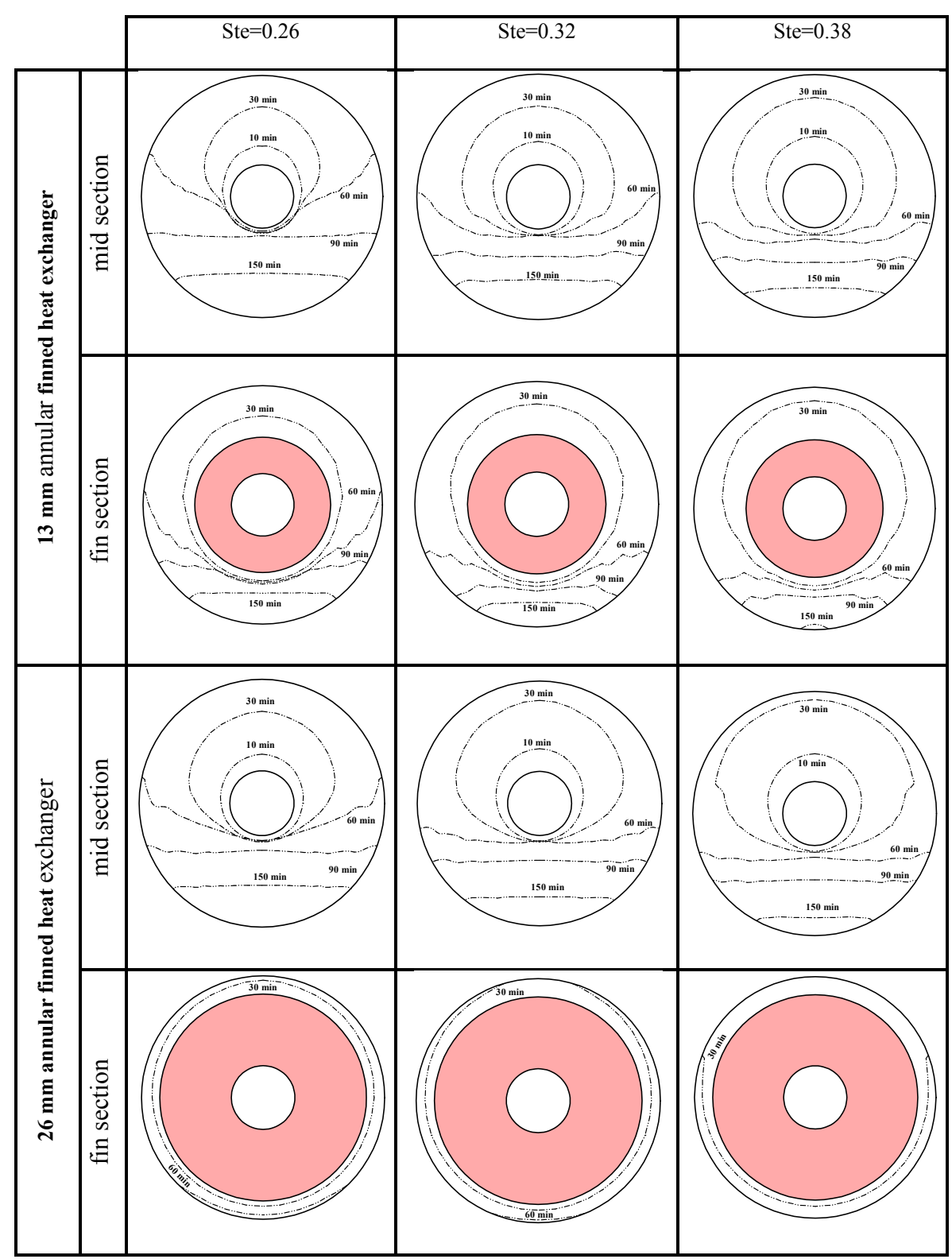

Figure 7. Numerical results showing melting front in shell for different Stefan numbers in 13 and 26 mm fins' height at mid section and fin section.

tices results in superior heat transfer. In this study, in order to investigate temperature variation in every point of these regions and to study the heat transfer mechanisms, local temperatures are recorded via precisely implemented thermocouples. Figure 5 illustrates the temperature distribution in every direction of the enhanced finned heat exchanger including radial, angular and axial for Stephan number of 0.26 and fins' height of $13 \mathrm{~mm}$ while the $26 \mathrm{~mm}$ counterpart is shown in Fig. 6.

For both of the heat exchangers, the sensor at angle of $0^{\circ}$ (above the central tube) senses a higher temperature regardless of time and section. This phenomenon is due to the upward movement of the hot front which originates from buoyancy law. Moreover, for the sections in which 6 thermocouples are employed, the lateral point $\left(90^{\circ}\right)$ is always again hotter than the underside one $\left(180^{\circ}\right)$ due to the same reason for both heat exchangers. Considering both figures, the interesting point here is that, while heating and before the emergence of melt drops, the temperature difference between 0 and $90^{\circ}$ is more considerable than this difference between 90 and $180^{\circ}$ however, when the melting process initiates and proceeds, the later temperature difference exceeds the first one. Comparing Figs. 5 and 6, it can be concluded that, utilizing higher fins due to farther radial penetration of 


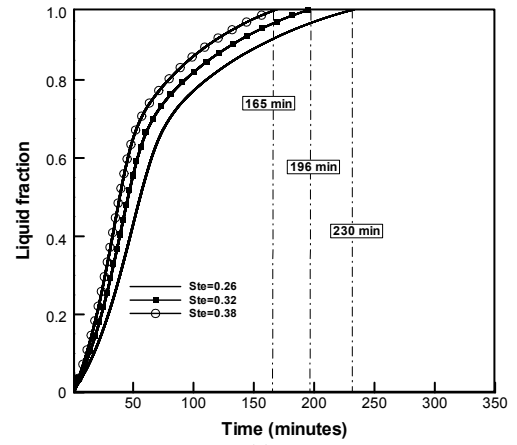

(a)

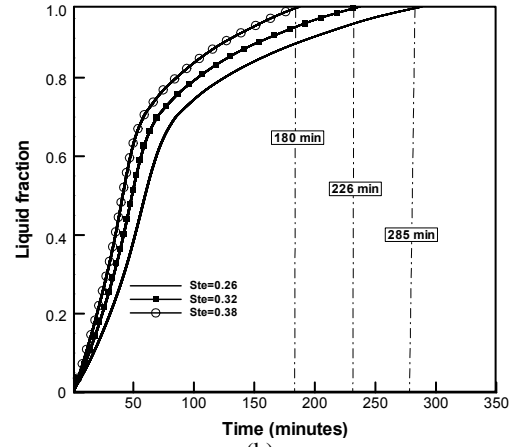

(b)

Figure 8. Numerical results showing liquid fraction variation versus time at different Stefan numbers: (a) 26 mm annular fin heat exchanger and (b) $13 \mathrm{~mm}$ annular fin heat exchanger.
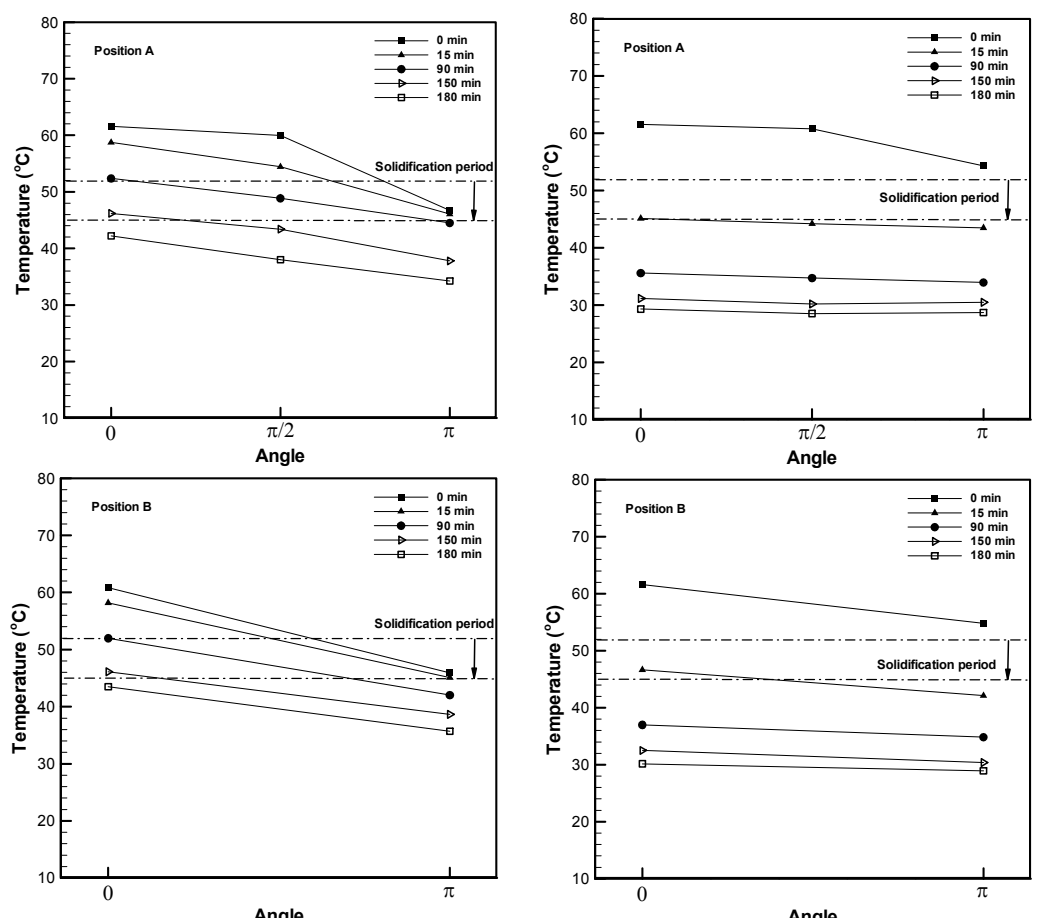

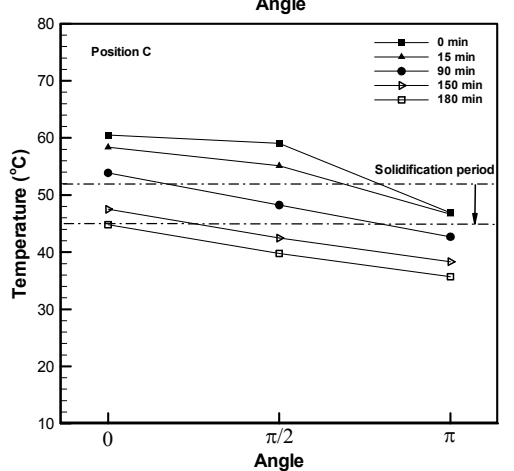

(a)

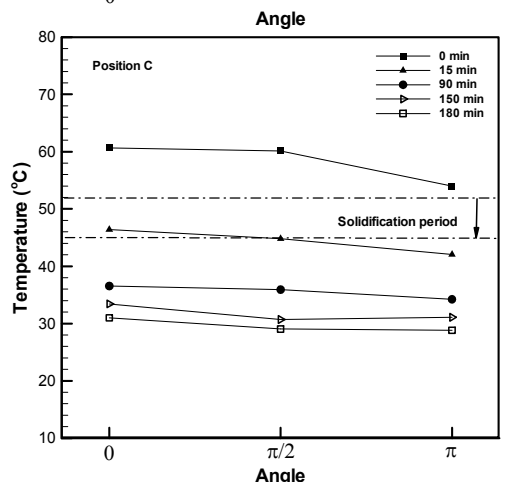

(b)

Figure 9. Experimental results of temperature distribution for $13 \mathrm{~mm}$ height fins heat exchanger for different angles during discharging process for Ste $=0.26$ : (a) $32.5 \mathrm{~mm}$ radial distance and (b) $12.5 \mathrm{~mm}$ radial distance. 

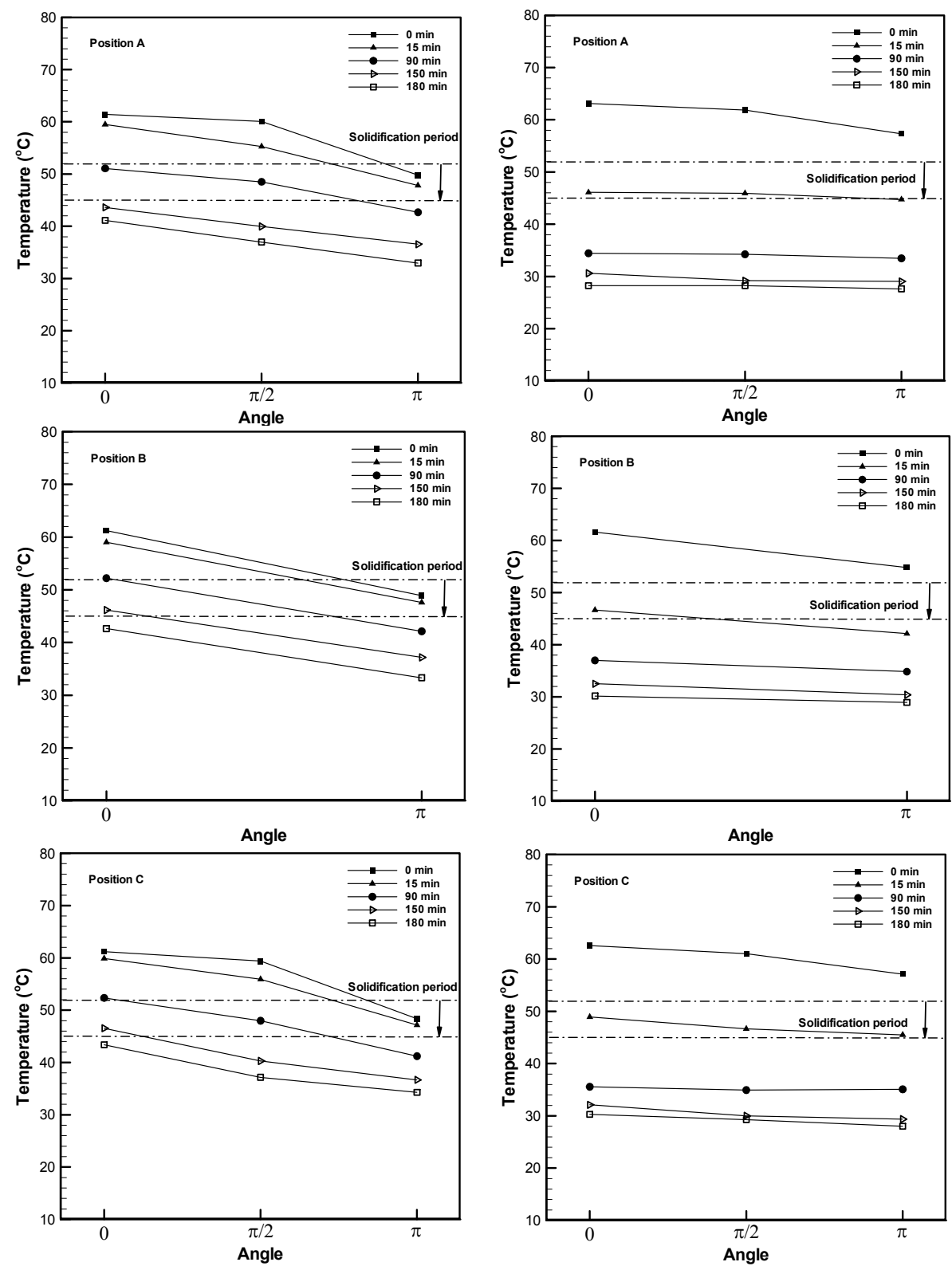

(a)

(b)

Figure 10. Experimental results of temperature distribution for $26 \mathrm{~mm}$ height fins heat exchanger for different angles during discharging process for $S t e=0.26$ : (a) $32.5 \mathrm{~mm}$ radial distance and (b) $12.5 \mathrm{~mm}$ radial distance.

heat during initial stages of melting process leads to an accelerated melting process which reduces the melting time. This statement may be verified by comparison of temperature and melting time for both heat exchangers at $32.5 \mathrm{~mm}$ radius of the three sections; $A, B$ and $C$.

In Fig. 7, melting front profiles are illustrated for the tube mid-section and the section of fin for both fins' height and varying Stephan numbers. Considering the profiles of the fin section, it can be seen that melting rate is much more accelerated in these sections in comparison with the mid-sections. The increase is so intense that, for Stephan number of 0.26 and after $60 \mathrm{~min}$ of experiment, almost no solid PCM was found around the fin. Implementation of higher fins of $26 \mathrm{~mm}$ not only, as stated, increases the rate of melting process at the fin section, but also it accelerates the process in all other sections in comparison with the heat exchanger enhanced with $13 \mathrm{~mm}$ fins. In addition, as the melting front is larger for the higher Stephan number (0.38), it is obvious that increasing this dimensionless number lessens the melting time. This effect is so significant that, a less amount of PCM remains in the shell in comparison with other Stephan numbers at a special time. 


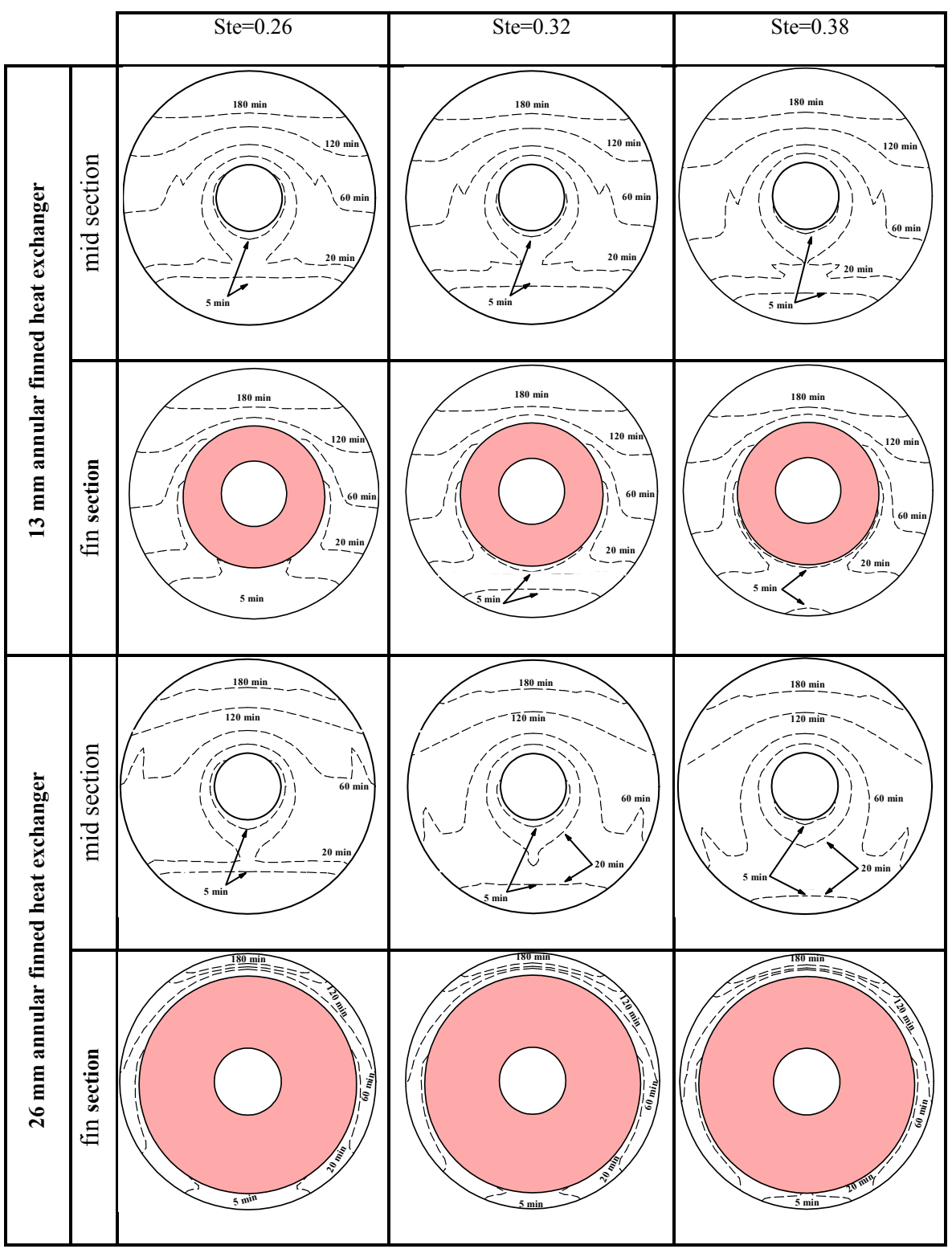

Figure 11. Numerical results of solid front in shell for different Stefan numbers in 13 and 26 mm fins' height at mid section and fin section.

Figure 8 shows the liquid fraction as a function of time. As can be seen in the figure, increasing Stephan number from 0.26 to 0.32 and then to 0.38 of $13 \mathrm{~mm}$-fin heat exchanger, the melting time diminishes 20 and 38 percent respectively. These reductions are 15 and 28 for $26 \mathrm{~mm}$-fin heat exchanger. Comparing Fig. $8 \mathrm{a}$ and $\mathrm{b}$ it can be implied that by increasing the fins' height from 13 to $26 \mathrm{~mm}$, the total melting time reduces up to $19 \%$.

\subsection{Discharging process}

In Figs. 9 and 10 temperature distribution are shown in radial, angular and longitudinal directions for Stefan number of 0.26 during discharge process. Comparing temperature values of these two, it can be figured out that, at the end of melting process for the $26 \mathrm{~mm}$ fin, temperature values are more than those of $13 \mathrm{~mm}$ fins heat exchanger. When time passes, the fins' presence play a more important role and consequently, the rate of temperature reduction exceeds that of $13 \mathrm{~mm}$ fins. In other words, the penetration of fins through the PCM results in less final discharge temperature which is more observable for farther radial distances of the cold carrying tube.

Figure 11 exhibits the solid front formation of the both heat exchangers through the discharge process for the midsection and the fin section, for different Stephan numbers. The solidification starts with a thin layer of PCM which covers the cold central tube of the heat exchanger. At the fin 
sections, due to the fin presence, the solidification process progresses more rapidly. As can be seen in the figure, for $13 \mathrm{~mm}$-fin heat exchangers, after $5 \mathrm{~min}$ of the experiment initiation, the solid front fills the bottom of the fin (at $180^{\circ}$ ). For the $26 \mathrm{~mm}$-fin heat exchanger, the coverage of $180^{\circ}$ is completed after $20 \mathrm{~min}$. This figure also shows that, while the Stephan number rises, although the solidification process begins later, the total solidification time stays almost identical for all Stephan numbers. The figure also implies that, at the fin's section, the solidification rate is higher due to the presence of fines. Comparing the solid formation process for both heat exchangers, it is seen that at initial stages of the process, due to the initial condition of higher temperature, the solid formation for the $26 \mathrm{~mm}$-fin heat exchanger occurs more slowly. However, after 60 min of the discharge, the doubling of the fins' height exhibit its influence and the growth of the solid front overtakes the heat exchanger with smaller fins' height. To quantify this phenomenon, $26 \mathrm{~mm}$-fin heat exchanger, for Stephan number of 0.26 , reduces the solidification time up to $16 \%$, comparing the $13 \mathrm{~mm}$-fin heat exchanger (from 460 to $384 \mathrm{~min}$ ).

\section{Conclusions}

In this investigation, two parameters including fins' height and Stefan number are studied for a double tube heat exchanger. The results show that:
- PCM temperature at above the tube, regardless of fin size and Stefan number is more than other angular direction around the tube.

- Studying temperature distribution, it is revealed that a point temperature of the PCM in the higher fin heat exchanger is more than the similar point in the shorter fins heat exchanger. This behavior is more obvious for farther radial regions.

- Increasing the Stefan number reduces the melting time for both heat exchangers.

- Increasing fins' height reduces the melting time which is more pronounced for greater Stefan numbers; increasing Stefan number from 0.26 to 0.38 results in melting time reduction to 8.3 and 19.2 respectively for the studied heat exchangers.

- Investigating solidification front, it is clear that, fin's height does not affect the front at mid-section at initial stages of the process progression. While the process advances, solidification front is more influenced by the fins' height in a way that at fins' section, the solidification front progresses in a much faster rate.

- Studying the effect of fins' size on melting and solidification processes, it can be seen that, higher fins influences the solidification time more than melting. 
Appendix A

Table A1. Nomenclatures.

\begin{tabular}{ll}
\hline$c_{p}$ & Specific heat capacity $\left(\mathrm{J} \mathrm{kg}^{-1} \mathrm{~K}^{-1}\right)$ \\
$g$ & Gravity $\left(\mathrm{m} \mathrm{s}^{-2}\right)$ \\
$h$ & Sensible enthalpy $\left(\mathrm{J} \mathrm{kg}^{-1}\right)$ \\
$H$ & Total enthalpy $(\mathrm{J})$ \\
$k$ & Thermal conductivity $\left(\mathrm{W} \mathrm{m}^{-1} \mathrm{~K}^{-1}\right)$ \\
$L$ & Latent heat $\left(\mathrm{Jg}^{-1}\right)$ \\
$\dot{m}$ & Mass flow rate $\left(\mathrm{kg} \mathrm{s}^{-1}\right)$ \\
$P$ & Pressure $(\mathrm{Pa})$ \\
$r$ & Radial distances from the center of HTF tube (m) \\
$S$ & Source term \\
$S t e$ & Stefan number \\
$T$ & Temperature $(\mathrm{K})$ \\
$V$ & Velocity vector $\left(\mathrm{m} \mathrm{s}^{-1}\right)$ \\
\hline & \\
\hline$\beta$ & $\quad$ Expansion coefficient $\left(\mathrm{K}^{-1}\right)$ \\
$\mu$ & Dynamic viscosity $(\mathrm{Pa} \mathrm{s})$ \\
$\lambda$ & Liquid fraction \\
$\rho$ & Density (kg m $\left.{ }^{-3}\right)$ \\
\hline & \\
\hline ch & Charge \\
mush & Mushy zone \\
$\mathrm{m}$ & Melting \\
ref & Reference \\
$\mathrm{H}$ & Hot water \\
Ini & Initial \\
Out & Outlet water \\
PCM & Phase change material \\
$\mathrm{S}$ & Solid \\
Liq & Liquid \\
\hline & \\
\hline &
\end{tabular}


Edited by: A. Barari

Reviewed by: two anonymous referees

\section{References}

Agyenim, F. and Hewitt, N.: The development of a finned phase change material (PCM) storage system to take advantage of offpeak electricity tariff for improvement in cost of heat pump operation, Energy Buildings, 42, 1552-1560, 2010.

Agyenim, F., Eames, P., and Smyth, M.: A comparison of heat transfer enhancement in a medium temperature thermal energy storage heat exchanger using fins, Solar Energy, 83, 1509-1520, 2009.

Agyenim, F., Eames, P., and Smyth, M.: Heat transfer enhancement in medium temperature thermal energy storage system using a multitube heat transfer array, Renewable Energy, 35, 198-207, 2010.

Agyenim, F., Eames, P., and Smyth, M.: Experimental study on the melting and solidification behavior of a medium temperature phase change storage material (Erythritol) system augmented with fins to power a $\mathrm{LiBr} / \mathrm{H}_{2} \mathrm{O}$ absorption cooling system, Renewable Energy, 36, 108-117, 2011.

Brent, A. D., Voller, V. R., and Reid, K. J.: Enthalpy-porosity technique for modeling convection-diffusion phase change: application to the melting of a pure metal, Numer. Heat Trans. B, 13, 297-318, 1988 .

Ezan, M. A., Ozdogan, M., and Erek, A.: Experimental study on charging and discharging periods of water in a latent heat storage unit, Int. J. Therm. Sci., 50, 2205-2219, 2011.

Gong, Z. X., Devahastin, S., and Mujumdar, A. S.: Enhanced heat transfer in free convection-dominated melting in a rectangular cavity with an isothermal vertical wall, Appl. Therm. Eng., 19, 1237-1251, 1999.

Hosseini, M. J., Ranjbar, A. A., Sedighi, K., and Rahimi, M.: A combined experimental and computational study on the melting behavior of a medium temperature phase change storage material inside shell and tube heat exchanger, Int. Commun. Heat Mass Trans., 39, 1416-1424, 2012.

Hosseini, M. J., Rahimi, M., and Bahrampoury, R.: Experimental and computational evolution of a shell and tube heat exchanger as a PCM thermal storage system, Int. Commun. Heat Mass Trans., 50, 128-136, 2014.

Ismail, K. A. R., Alves, C. L. F., and Modesto, M. S.: Numerical and experimental study on the solidification of PCM around a vertical axially finned isothermal cylinder, Appl. Therm. Eng., 21, 53-77, 2001.
Liu, C. and Groulx, D.: Experimental study of the phase change heat transfer inside a horizontal cylindrical latent heat energy storage system, Int. J. Therm. Sci., 82, 100-110, 2014.

Mat, S., Al-Abidi, A. A., Sopian, K., Sulaiman, M. Y., and Mohammad, A. T.: Enhance heat transfer for PCM melting in triplex tube with internal-external fins, Energy Convers. Manage., 74 223-236, 2013.

Mehling, H. and Cabeza, L.: Phase change materials and their basic properties, in: Thermal Energy Storage for Sustainable Energy Consumption, edited by: Paksoy, H. Ö., Springer, Netherlands, 257-277, 2007.

Mosaffa, A. H., Talati, F., Tabrizi, H. B., and Rosen, M. A.: Analytical modeling of PCM solidification in a shell and tube finned thermal storage for air conditioning systems, Energy Buildings, 49, 356-361, 2012.

Ogoh, W. and Groulx, D.: Effects of the number and distribution of fins on the storage characteristics of a cylindrical latent heat energy storage system: a numerical study, Int. J. Heat and Mass Trans., 48, 1825-1835, 2012.

Pakrouh, R. Hosseini, M. J., and Ranjbar, A. A.: A parametric investigation of a PCM-based pin $n$ heat sink, Mechanical Science, 6, 65-73, 2015.

Patankar, S. V.: Numerical Heat Transfer and Fluid Flow, Hemisphere, Washington, D.C., 1980.

Rahimi, M., Ranjbar, A. A., Ganji, D. D., Sedighi, K., Hosseini, M. J., and Bahrampoury, R.: Analysis of geometrical and operational parameters of PCM in a fin and tube heat exchanger, Int. Commun. Heat Mass Trans., 53, 109-115, 2014.

Seeniraj, R. V. and Narasimhan, N. L.: Performance enhancement of a solar dynamic LHTS module having both fins and multiple PCMs, Solar Energy, 82, 535-542, 2008.

Sharma, A., Tyagi, V. V., Chen, C. R., and Buddhi, D.: Review on thermal energy storage with phase change materials and applications, Renewable Sustain. Energy Rev., 13, 318-345, 2009.

Shatikian, V., Ziskind, G., and Letan, R.: Numerical investigation of a PCM-based heat sink with internal fins, Int. J. Heat Mass Trans., 48, 3689-3706, 2005.

Zalba, B., Marin, J. M., Cabeza, L. F., and Mehling, H.: Review on Thermal Energy Storage with Phase Change Materials, Heat Transfer Analysis and Applications, Appl. Therm. Eng., 23, 251283, 2003. 